\title{
Robust Gloves For 3D Interaction In Mobile Outdoor AR Environments
}

\author{
Wayne Piekarski and Ross Smith \\ Wearable Computer Laboratory \\ School of Computer and Information Science \\ University of South Australia \\ Mawson Lakes, SA, 5095, Australia \\ wayne@cs.unisa.edu.au, ross@cs.unisa.edu.au
}

\begin{abstract}
This paper describes the design of hand-worn gloves for interacting with mobile outdoor augmented reality systems. Most existing systems rely on more traditional $2 D$ input devices such as mice and keyboards. Since augmented reality information is typically registered in $3 D$ to the environment, user interfaces need to be designed that are capable of supporting the more complex operations possible. This paper describes how we used metallic thread and adhesive fabric to add conduction sensing to a standard set of gloves which can survive harsh treatment; how Bluetooth and MSP430 microcontrollers are used to build a small circuit that is wireless and highly portable; and how ARToolKit is used for $3 D$ tracking of fiducial markers on the thumbs. While we have previously demonstrated this technology with a number of our previous systems, this paper explains the various techniques we use in the implementation.
\end{abstract}

\section{INTRODUCTION}

We have been performing a wide range of research into developing 3D applications for use in mobile outdoor augmented reality (AR) environments. Since these environments are 3D in nature, traditional input devices such as mice and keyboards are not necessarily suitable for use. We have therefore been using tracked gloves as an input device for many of our applications, such as the previously presented Tinmith-Metro 3D modelling application [6]. Gloves have been used as input devices in a large number of VR systems; they allow the use of the hands to interact with the environment using natural pinch and grab style gestures. There are a number of commercially available gloves on the market and these perform sensing in two different ways: the FakeSpace PinchGlove [1] contains electrical contact sensors at each fingertip to measure finger pinches, while the VTi CyberGlove [7] uses bend sensors to measure finger positions. While full motion of the fingers may seem more accurate, errors in the results make it difficult for software to determine exactly when the user's fingers are pressed together. With our user interface only requiring simple finger press inputs, a pinch-style design is most desirable for accuracy. However, these commercial gloves cannot be used because the controller unit is not portable and the gloves require cables to connect up with. This paper presents a new glove design that can provide 6DOF tracking outdoors, is totally wireless, uses robust wiring, supports custom pad layouts, and has extremely low power requirements. This glove design is shown in Figure 1 from both sides of the user's hands.

\section{GLOVE CONSTRUCTION}

The detection of finger presses in this design relies on the use of conductive fabric pads with adhesive to fix them to the fingertips of standard gloves. While other gloves such as FakeSpace PinchGloves can only measure fingers pressing against the thumbs, ours support presses against the palms as well. If desired, extra pads could also be added to other locations on the hand as well. When a voltage is applied by the controller to one of the fingers, it tests to see if that voltage is conducted through to the sensing pads on the thumbs or the palm. This is a simple yet effective way of testing what a finger is pressing against. The controller needs to send and receive voltages from the various pads, and so conductive thread has been sewn into the seams of the gloves. During construction, a needle is used to route the thread internally through the glove seams from the fingertips to the controller. Previously we used wires attached on the outside with glue, but these wires were easy to break and were also quite rigid. In contrast, with the conductive thread being strong and flexible just like standard thread, the wiring is highly robust and undetectable to the user when sewn into the seams. An interesting problem is that since the conductive thread comes into contact with the wearer's skin, sometimes it is possible for signals to conduct along the fingers and generate false readings. By testing the signal stability and voltage level in the microcontroller, it is possible to detect these cases of skin conduction and filter them out. Any type of glove that the user prefers to use is suitable for modification, as the adhesive pads and conductive thread are all that needs to be added. Gloves that have fabric which is more rigid and not so stretchable is desirable, to prevent the metallic thread from moving too much inside the seams.

\section{CONTROLLER DESIGN}

Using the main host computer to perform the detection of the finger presses of the gloves is quite time consuming and will waste a considerable amount of its resources to do this. As a result, offloading the processing to a dedicated microcontroller is desirable. Furthermore, with the availability of tiny Bluetooth implementations such as the Promi-SD202 chip, it is possible to add Bluetooth RFCOMM support to any existing system that

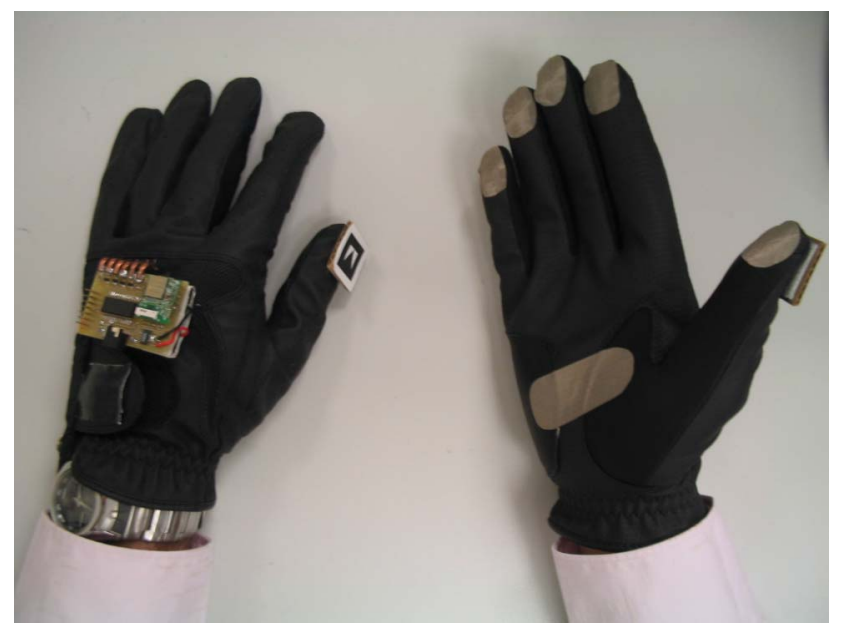

Figure 1 - The gloves shown from above and underneath. A custom controller board with an MSP430 and Promi-SD202 Bluetooth chip is used to interface with the gloves. Metallic fabric pads and conductive thread are used to detect finger presses. Fiducial markers are used for 3D tracking outdoors. 
uses RS-232. We designed a custom controller board that contains a Texas Instruments MSP430, a 3.7V 850mAh Lithium polymer battery, voltage regulator circuits, and a Promi-SD202 Bluetooth chip. The MSP430 is responsible for sending the voltage pulses to each of the fingers hundreds of times every second, and then determining if contact is made with either the thumb or the palm. Since the polling speed is so fast, debouncing code is possible to filter out any glitches that occur when the contact is made. Simple text strings are then sent via the serial port and transmitted via Bluetooth to the host PC, so that applications can interpret the presses and releases of the gloves. The host PC is able to dynamically discover Bluetooth controller chips nearby, and then connect using the RFCOMM protocol to easily provide a virtual serial port for applications. The power consumption of the MSP430 is negligible compared to the Bluetooth chip, and the circuit can run for days without recharging. The MSP430 disables the operation of the Bluetooth chip if it detects that the battery level has fallen too low, protecting the battery from dangerously low discharge levels.

\section{FidUCIAL MARKER TRACKING}

3D tracking of the hands is used to perform a wide-range of tasks in our AR applications, and performing tracking outdoors suffers from a number of environmental problems such as power consumption, size, weight, and support infrastructure. The outdoor environment also contains harsh conditions that cannot be controlled by the user, and makes many tracking technologies unusable: accelerometers drift over time and the registration would become inadequate within a few seconds; infrared-based systems are vulnerable to the wide-range of infrared light given off by the sun; and active magnetic tracking relies on large nonportable control units that are possibly also interfered with by the other electronics in our mobile AR system.

One solution demonstrated outdoors was the WearTrack system [2] by Foxlin and Harrington that utilised ultrasonic technology. This system tracked the position of the user's hand relative to their head (with no orientation), and was used for a number of interaction tasks. This required the addition of an ultrasonic tracker with its associated weight, size, and electric power penalties that must be added to the wearable computer system.

The glove design presented here uses an optically-based vision tracking system, leveraging the existing helmet mounted camera that is currently used for the live AR video overlay. The ARToolKit libraries [3] are employed to perform full six degree of freedom tracking of the fiducial markers placed on the thumbs of the gloves. Since ARToolKit provides 6DOF tracking, it can provide information about the position and orientation of the hands in real-time. The registration of the tracking is always excellent since the video seen by ARToolKit is the same as is used to provide the AR video overlay to the user. On the gloves, simple $2 \mathrm{~cm} \times 2 \mathrm{~cm}$ paper fiducial markers are glued onto cardboard and then attached to the thumbs of the glove. It should be noted that the ARToolKit calibration process can introduce errors of its own, and these will affect the accuracy of the tracking achieved. We have published an earlier paper that discusses how to correct some of these errors to achieve higher accuracy [5].

It was initially proposed to put the fiducial markers on the back of the user's hands, but it was quickly realised that the hands are large and fill much of the field of view of the camera. A user would not be able keep the markers in the field of view and manipulate an object to the edge of the display. The problem of accurately calculating the user's finger-tip position based on fiducial markers on the wrists was also a concern. The ends of the fingers were also dismissed, as it was found that they moved too much during menu option selections and would affect operations in progress. It was noticed however that the thumb does not move much during pinch motions, and so this is an ideal location to place the fiducial marker. The user can then hold their hands out in front of them with a comfortable stance to perform the desired tasks, and in a way that is visible to the head-mounted camera.

When in use outdoors, we found that the varying lighting conditions caused ARToolKit to fail in its tracking. This is because the required threshold value can vary considerably depending on the conditions, and there is no one ideal value for all situations. We therefore use a randomised threshold algorithm as proposed by the Studierstube Handheld AR group [8], where if the tracking fails then a random value is selected for the next frame. At a frame rate of 30 FPS, the tracking resumes itself within less than a fraction of a second under the most troublesome of conditions. We have found that the reliability of the tracker has improved significantly with this. We have also previously performed studies into the use of ARToolKit [4] for tracking, and found that its accuracy varies depending on the rotation of the marker relative to the camera. Also, the ability to overlay a cursor on the marker is more accurate than the ability to measure rotation. In cases where high quality rotations are required, we use two-handed techniques to derive rotation from position.

\section{Conclusion}

This paper has presented the novel design for a set of gloves that are wireless, low powered, and able to support 6DOF tracking in mobile outdoor environments. These environments are particularly harsh on hardware but also tracking, and this design has been used for a number of years in supporting 3D user interfaces with excellent results.

\section{REFERENCES}

[1] FakeSpace Labs. Pinch Gloves http://www.fakespacelabs.com/products/pinch.html

[2] Foxlin, E. and Harrington, M. WearTrack: A SelfReferenced Head and Hand Tracker for Wearable Computers and Portable VR. In 4th Int'l Symposium on Wearable Computers, pp 155-162, Atlanta, Ga, Oct 2000.

[3] Kato, H. and Billinghurst, M. Marker Tracking and HMD Calibration for a Video-based Augmented Reality Conferencing System. In 2nd Int'l Workshop on Augmented Reality, pp 85-94, San Francisco, Ca, Oct 1999.

[4] Malbezin, P., Piekarski, W., and Thomas, B. H. Measuring ARToolKit Accuracy in Long Distance Tracking Experiments. In 1st Int'l Augmented Reality Toolkit Workshop, Darmstadt, Germany, Sep 2002.

[5] Piekarski, W. and Thomas, B. H. Using ARToolKit for 3D Hand Position Tracking in Mobile Outdoor Environments. In 1st Int'l Augmented Reality Toolkit Workshop, Darmstadt, Germany, Sep 2002.

[6] Piekarski, W. and Thomas, B. H. Interactive Augmented Reality Techniques for Construction at a Distance of 3D Geometry. In 7th Int'l Workshop on Immersive Projection Technology / 9th Eurographics Workshop on Virtual Environments, Zurich, Switzerland, May 2003.

[7] Virtual Technologies. CyberGlove. http://www.virtex.com/products/hw_products/cyberglove.html

[8] Studierstube Handheld AR Group. http://www.studierstube.org/handheld_ar 